vidui senza milza comprende: la vaccinazione, la profilassi antibiotica e l'informazione dei pazienti.

Tuttavia, nonostante sia molto importante ridurre il rischio di sepsi in tali pazienti, in pratica queste misure preventive non vengono quasi mai proposte. Infatti, il nostro paziente, non consapevole di essere un individuo ad alto rischio di sepsi, aveva ignorato i pochi segnali che la polmonite pneumococcica aveva dato. E' molto importante allora, coinvolgere i pazienti sul rischio settico dopo la splenectomia soprattutto con l'informazione (e l'aiuto del clinico); inoltre, nel caso di una sospetta sepsi, è fondamentale l'anamnesi del paziente per improntare un percorso diagnostico mirato, soprattutto in urgenza.

020

\section{VALUTAZIONE DEL NUOVO SISTEMA AUTOMATICO “VIDIA" PER LA RICERCA DI ANTICORPI ANTI TOXOPLASMA E ROSOLIA}

\author{
Ferraironi M., Martinelli D. e Cipriani P.
}

Il Facoltà di Medicina e Chirurgia Università La Sapienza Roma - Servizio di Microbiologia, Azienda Ospedaliera S. Andrea, via di Grottarossa 1035, 00189 Roma

Introduzione. Il sistema VIDIA (bioMérieux) è un nuovo strumento automatico con gestione della provetta primaria, concepito per semplificare la routine e per implementare il livello di rintracciabilità dei dati.

Nel presente lavoro abbiamo valutato le performance dei test VIDIA per gli anticorpi anti-Toxoplasma ed anti-Rosolia confrontandola con il sistema attualmente in uso nel nostro laboratorio AxSym - Abbott.

Metodi. Sono stati analizzati complessivamente 200 campioni appartenenti ad una popolazione mista, per determinare TOXO IgG e IgM, e RUB IgG e IgM. I campioni con risultati discordanti sono stati ripetuti con il metodo VIDA e, inoltre, sono previsti ulteriori approfondimenti utilizzando il test di riferimento Toxo Isaga.

È stata inoltre valutata la ripetibilità intra-serie e la riproducibilità inter-serie del test VIDIA TOXO IgG e VIDIA RUB IgG secondo i suggerimenti dell'NCCLS, utilizzando 2 pool di sieri a 4 diverse concentrazioni.

Risultati. Globalmente si è riscontrata una prevalenza pari a circa il $19 \%$ per Toxo IgG, il 3\% per Toxo IgM, il $77 \%$ per Rosolia IgG e 1' 1\% per Rosolia IgM.

La concordanza tra i metodi a confronto è stata del 100\% per il Toxo IgG, 98\% per Toxo IgM, 99\% per Rosolia IgG e 99\% per Rosolia IgM.

Sui risultati discordanti sono in corso approfondimenti diagnostici per stabilire se tale discordanza sia da imputare solo al metodo usato o possa dipendere anche dallo stato immunologico dei pazienti soprattutto se si tratta di donne in gravidanza.

La precisione intra-serie e la ripetibilità inter-serie $(\mathrm{CV} \%)$ dei test VIDIA TOXO IgG e VIDIA RUB IgG erano comprese tra 2 e $4 \%$ circa.

Conclusioni. Dai risultati emerge un elevato livello di concordanza tra il sistema VIDIA e il metodo AxSym attualmente in uso. Si rileva inoltre un elevatissimo grado di precisione intra-serie ed inter-serie dei test VIDIA.

\section{1}

\section{LEPTOSPIROSI CON ISOLAMENTO COLTURALE POSITIVO}

\author{
Franzin L.', Cabodi D.', Ciarrocchi S. ${ }^{2}$,
}

Pinto A. ${ }^{2}$, Ciceroni L. ${ }^{2}$

' Laboratorio "Ricerca Speciale Microbiologica",

Dipartimento Diagnostica di Laboratorio,

Ospedale Amedeo di Savoia,

Corso Svizzera 164, 10149 Torino.

${ }^{2}$ Centro Nazionale per le Leptospirosi,

Dipartimento di Malattie Infettive, Parassitarie

ed Immunomediate, Istituto Superiore di Sanità,

Viale Regina Elena 299, 0016 I Roma.

Introduzione. Leptospirosi è una malattia di difficile diagnosi di laboratorio. L'esame colturale fornisce una prova diretta e definitiva dell'infezione, non sempre ottenibile con esami sierologici. Nella leptospirosi a rapido decorso ed esito infausto la coltura è l'unico metodo in grado di consentirne la diagnosi. Inoltre la tipizzazione dei ceppi isolati è di rilevante importanza epidemiologi$\mathrm{ca}$, in quanto permette di identificare il serovar infettante, di conoscerne la distribuzione geografica e talvolta di stabilire la sorgente di infezione o il reservoir animale. Riportiamo un caso di leptospirosi fulminante, diagnosticato con coltura, per il quale è stato definito il serovar infettante mediante tecniche di tipizzazione sierologica e molecolare.

Metodi. Paziente: soggetto maschio di 35 anni con febbre, ittero, insufficienza renale acuta, shock cardiocircolatorio. Circostanza presunta del contagio: caduta accidentale con automobile in un canale. Comparsa dei sintomi: una settimana dopo l'incidente. Penicillina G è stata somministrata dal $4^{\circ}$ giorno dall'inizio dei sintomi. Il paziente è deceduto il giorno successivo. Metodi: La ricerca di anticorpi è stata eseguita su siero prelevato al $4^{\circ}$ giorno di malattia mediante la reazione di agglutinazione microscopica (MAT) eseguita con 20 ceppi rappresentativi di 19 serovar e 14 sierogruppi. L'esame colturale è stato eseguito da sangue eparinato, da urina e da campioni autoptici (rene, polmone, milza, miocardio, fegato). Risultati. La sierodiagnosi ha evidenziato anticorpi antiLeptospira 1:320 verso il serovar Poi. La coltura è risultata positiva da sangue e da urina. Entrambe gli isolati sono stati identificati come serovar Icterohaemorrhagiae (sierogruppo Icterohaemorrhagiae) mediante MAT eseguita con antisieri di coniglio ed anticorpi monoclonali. I risultati della tipizzazione sierologica sono stati confermati mediante analisi del DNA genomico eseguita con PFGE.

Conclusioni. I risultati ottenuti confermano l'importanza dell'esame colturale nella diagnosi di leptospirosi e che solo attraverso la tipizzazione degli isolati è possibile identificare con certezza il serovar di appartenenza della leptospira infettante. 